Flores-Aguilar, G.; Fernández-Río, J.; Prat-Grau, M. (2021) Gamificating Physical Education Pedagogy. College Students' Feelings. Revista Internacional de Medicina y Ciencias de la Actividad Física y el Deporte vol. 21 (84) pp. 515-533. Http://cdeporte.rediris.es/revista/revista83/artgamificando1274.htm

DOI: https://doi.org/10.15366/rimcafd2021.83.007

\title{
ORIGINAL
}

\section{GAMIFICANDO LA DIDÁCTICA DE LA EDUCACIÓN FÍSICA. VISIÓN DEL ALUMNADO UNIVERSITARIO}

\section{GAMIFICATING PHYSICAL EDUCATION PEDAGOGY. COLLEGE STUDENTS' FEELINGS}

Flores-Aguilar, G. ${ }^{1}$; Fernández-Río, J. y Prat-Grau, M. ${ }^{3}$

${ }^{1}$ Doctor en Didáctica de la Educación Física. Facultad de Ciencias de la Educación. Universidad de Sevilla (España) gfaquilar@us.es

2 Doctor en Pedagogía. Facultad de Formación del Profesorado y Educación. Universidad de Oviedo (España) javier.rio@uniovi.es

3 Doctora en Pedagogía. Facultad de Ciencias de la Educación. Universidad Autónoma de Barcelona (España) maria.prat@uab.cat

Código UNESCO I UNESCO code: 5803.02 Preparación de profesores / Preparing teachers

Clasificación Consejo de Europa / Council of Europe classification: 5 Didáctica y metodología / Didactics and methodology

Recibido 18 de julio de 2019 Received july 19, 2919

Aceptado 3 de diciembre de 2019 Accepted december 3, 2919

\section{RESUMEN}

El objetivo de este trabajo fue valorar el impacto de la experiencia gamificada "Súper Mario Bros" en la formación inicial universitaria de futuros docentes de Educación Física. Los 76 estudiantes matriculados en la asignatura "Didáctica de la Educación Física II" del Grado de Ciencias de la Actividad Física y del Deporte de una universidad del Este de España accedieron a participar. Todos respondieron a un cuestionario online ad hoc compuesto por preguntas abiertas y cerradas (escala Likert). Los resultados revelaron percepciones muy positivas y satisfactorias sobre la experiencia en general, y de sus pilares educativos en particular; destacándose, por encima de todo, una incidencia directa en el grado de motivación y compromiso hacia la asignatura; además del deseo de modificar la creación de grupos heterogéneos.

PALABRAS CLAVE: Gamificación, aprendizaje cooperativo, didáctica de la educación física, evaluación formativa. 


\section{ABSTRACT}

The aim of the study was to assess the impact of the Gamificated experience "Super Mario Bros" in future Physical Education teachers' initial training. All students enrolled in the subject "Physical Education Didactics II" $(n=76)$ of the Undergraduate program in Physical Activity and Sport Sciences from a university in eastern Spain agreed to participate. The students answered an online ad hoc questionnaire, which included open and closed questions (Likert scale). Results revealed very positive and satisfactory perceptions of the experience and its structural characteristics; highlighting, above all, a direct impact on the students' motivation and commitment to the class; in addition to the desire to modify the formation of heterogeneous groups.

KEY WORDS: Gamification, cooperative learning, didactics of physical education, formative assessment.

\section{INTRODUCCIÓN}

La educación física (EF) del siglo XXI necesita ser replanteada en numerosos aspectos, y uno de ellos es su planteamiento de enseñanza (López-Pastor, Pérez, Manrique y Monjas, 2016). En este sentido, y para que el alumnado pase a ser el principal protagonista de su propio aprendizaje, la incorporación de las metodologías activas y/o los modelos pedagógicos en las clases de EF adquieren un especial interés (Haerens, Kirk, Cardon y De Bourdeaudhuij, 2011). Como paso previo, creemos que la formación del profesorado de EF tiene un rol importante a la hora de analizar, promocionar e incorporar estos nuevos planteamientos metodológicos en los actuales planes de estudios. De hecho, con la llegada del Espacio Europeo de Educación Superior (EEES), esto se convirtió en una necesidad de primera orden (Berné, Lozano y Marzo, 2011).

En base a estas ideas, en la asignatura de "Didáctica de la EF II", impartida en el tercer curso del Grado de Ciencias de la Actividad Física y del Deporte (CAFE), se ha llevado a cabo la experiencia "Súper Mario Bros" (Flores-Aguilar, 2019), donde la gamificación se hibrida con el aprendizaje cooperativo (AC) en el marco de la evaluación formativa. A continuación, un breve apunte teórico de los tres pilares de dicha experiencia.

\section{Gamificación}

También conocida como ludificación, ambos conceptos provienen del anglicismo Gamification (Kapp, Blair y Mesch, 2014). Aunque este término surge en el año 2008, no es hasta el 2010 cuando se empieza a generalizar en la educación y en otros ámbitos como el empresarial, la salud, el marketing, etc. (Melchor, 2012). A nivel general, gamificar consiste en vivir experiencias de juego en entornos no lúdicos (Werbach y Hunter, 2012). En esta línea, en el ámbito educativo la gamificación se considera una "metodología donde se utiliza una historia o narrativa imaginaria, como hilo conductor, con el fin de consolidar las competencias u objetivos de aprendizaje a partir de la introducción de las 
mecánicas de los juegos o videojuegos" (Flores-Aguilar, 2019, p.529). De hecho, y metafóricamente hablando, podría decirse que la gamificación surge como antídoto para curar los virus que últimamente rondan la educación (desmotivación, aburrimiento y falta de compromiso), a partir de las dinámicas, mecánicas, y componentes de los juegos (Llopis y Balaguer, 2016; Werbach y Hunter, 2012). Como dicen Cortizo, Carrero, Monsalve, Velasco, Díaz y Pérez (2011) y Monguillot, Arévalo, Mon, Batet y Catasús (2015), las reglas y los retos que aquí se crean generan un alto grado de compromiso y motivación en sus participantes. A su vez, Hanus y Fox (2015), Pérez-López y Rivera García (2017), entre otros, insisten en que el alumnado suele involucrarse de una forma más intensa, ya que se motiva más debido a sus sentimientos y emociones positivas. Cortizo et al. (2011) añaden que las dinámicas lúdicas fomentan la colaboración y la resolución de problemas, y disminuyen en el alumnado el miedo a equivocarse; no obstante, Carrasco, Matamoros y Flores-Aguilar, (2019) y Chan, Fui-Hoon, Liu y Lu, (2018) alertan de que no siempre se consiguen mejoras significativas entre el alumnado. Esta aparente contradicción señala que es necesaria más investigación sobre el fenómeno de la Gamificación en Educación. Melchor (2012) afirma que las experiencias gamificadas resultan exitosas si son atractivas y despiertan el interés del alumnado; y aquí juega un papel clave las categorías de la motivación intrínseca descritas por la teoría de la autodeterminación de Deci y Ryan (1985). En este sentido, Fernández-Rio y Flores-Aguilar (2019) señalan que la gamificación debe promover un clima de clase de maestría (el objetivo debe ser realizar bien la tarea sin preocuparse por superar o ganar a otros), donde se desarrolla la competencia (ver que se adquieren aprendizajes, y por eso la importancia de la evaluación formativa y su retroalimentación inmediata), las relaciones sociales (que se crean a través de los equipos cooperativos creados) y la autonomía (la necesidad de generar momentos donde los jugadores-alumnos tomen sus propias decisiones). En caso contrario, Pérez-Pueyo y Hortigüela (2020) alertan de la existencia de unas inadecuadas experiencias gamificadas, centradas en la incorporación de un indiscriminado número de recompensas que favorecen la competitividad individual y grupal, y que sólo inciden en la motivación extrínseca del alumnado, cuyo mantenimiento es a corto plazo. En base a estas premisas, y en la búsqueda de un cambio de actitudes en el alumnado, en este caso hacia su mirada hacia la EF y su Didáctica, la gamificación en educación debería entenderse como un modelo pedagógico que debería hibridarse, casi obligatoriamente, con el AC; y todo bajo el paraguas de la evaluación formativa (Fernández-Río y Flores-Aguilar, 2019), tal y como veremos posteriormente.

Finalmente, y aunque actualmente España es el segundo país con más publicaciones activas (Web of Science) sobre la temática (Kocakoyun y Ozdamli, 2018), las experiencias publicadas sobre Gamificación en las principales bases de datos nacionales e internacionales sobre el área de la EF y la formación del profesorado siguen siendo aún incipientes, sobre todo en cuanto al número de investigaciones se refiere (Pérez-López, Rivera y Trigueros, 2017; 2019); sirvan de ejemplo las recientes revisiones bibliográficas realizadas por León, MartínezMuñoz y Santos-Pastor (2019) y Escarvajal y Martín (2019) donde se identifican las principales publicaciones existentes, las cuales en su mayoría son experiencias docentes. 


\section{Aprendizaje cooperativo}

El AC es un modelo pedagógico donde el alumnado y el profesorado implicado aprende y co-aprende a partir de un planteamiento de enseñanza y aprendizaje que potencia su interacción grupal y su interdependencia positiva (FernándezRío, 2014). Según Velázquez (2015) son varios los estudios que coinciden en destacar al AC como eje clave para potenciar el aprendizaje motor y académico del alumnado, además de para la consolidación de conductas prosociales y el fomento de la inclusión y la valoración de la diversidad en las clases de EF (Fernández-Río y Méndez-Giménez, 2016). De igual modo, los estudios de Goodyear, Casey y Kirk (2014) señalan un ligero aumento de la motivación y el compromiso de los estudiantes que lo vivencian. Este proyecto pretende dar un poco más de luz a este vacío científico y por ello se apuesta por aplicar el AC a partir de los elementos básicos de Johnson, Johnson y Holubec (2013) como la interdependencia positiva (el trabajo de cada alumno es imprescindible para el beneficio del resto de compañeros y viceversa), la interacción promotora cara a cara (los estudiantes interactúan durante las tareas para ayudarse mutuamente), la responsabilidad individual (cada estudiante es responsable y tiene el compromiso de aportar sus tareas), el procesamiento grupal (la información que tiene el grupo debe ser procesada por cada miembro del grupo para su utilización) y las habilidades sociales (con el contacto directo los estudiantes mejoran sus habilidades interpersonales); además de algunas de sus técnicas más comunes (Resultado Colectivo y Piensa-Comparte-Actúa) (Orlick, 1982; Grineski, 1996). Como ya se ha señalado anteriormente, la investigación parece indicar que el AC puede proporcionar un marco de trabajo en el contexto de la Gamificación que puede ayudar a crear un clima de aula orientado a la tarea (Fernández-Rio y Flores-Aguilar, 2019), pero es necesaria más investigación para corroborarlo.

\section{Evaluación formativa en educación superior}

El aprendizaje real o significativo depende, en gran parte, de la evaluación (Gibbs, 2003). Antes que nada, cabría recordar que evaluar no es calificar. Ambos conceptos suelen confundirse, y esto sigue provocando nefastos efectos en el alumnado (López-Pastor, 2009; Palacios, López-Pastor y Barba, 2013). Como afirman Martínez y Flores-Aguilar (2014), la mayoría de las prácticas evaluadoras continúan limitándose a realizar una valoración final y sumativa. Dentro de las clasificaciones existentes, la evaluación formativa y compartida se reafirma como la más adecuada, ya que su propósito es el de optimizar el proceso de enseñanza-aprendizaje a partir de la transparencia (el alumnado sabe de primera mano qué, cómo y cuando se le avaluará), de la participación del alumnado (se crean espacios de diálogo, reflexión y decisión), y de los feedbacks que se generan (retroalimentación constante de los resultados) (Barrientos y López-Pastor, 2015; López-Pastor, 2009), entre otros aspectos.

Ante el cambio que conllevó la adaptación al Espacio Europeo de Educación Superior (EEES), la evaluación formativa se convirtió en uno de los ejes centrales del cambio (Martínez y Flores-Aguilar, 2014). Ejemplo de ellos son los múltiples trabajos que han estudiado y aplicado con éxito este sistema de evaluación en los estudios de formación del profesorado, sobre todo de EF (Castejón, López- 
Pastor, Julián y Zaragoza, 2011; Gallardo y Carter, 2016; López-Pastor, Pérez, Barba y Lorente, 2016). Y es que, tal y como revela la revisión de la literatura, la evaluación formativa mejora las competencias profesionales del futuro profesorado, su grado de implicación en las materias (Álvarez, Grau y Tortosa, 2010) y, por ende, su rendimiento académico suele verse influido positivamente (Montero, Villalobos y Valverde, 2007). En palabras de Boud y Falchikov (2007) y Rodríguez y Herrera (2009), la evaluación formativa ayuda a generar un elevado nivel de responsabilidad, implicación, participación y control sobre el propio aprendizaje del alumnado; gracias también a la introducción de procesos autoevaluadores y coevaluadores (Castejón et al., 2011).

\section{OBJETIVOS}

El objetivo principal de este trabajo fue conocer la visión del alumnado participante sobre el conjunto de la experiencia gamificada "Súper Mario Bros", en base a sus propias experiencias vividas durante el transcurso de una asignatura. Más concretamente, se quiso ahondar en sus percepciones y valoraciones sobre: a) los efectos de la experiencia en la motivación, el compromiso, el rendimiento y el aprendizaje; b) las características básicas de los pilares didácticos: gamificación, AC y evaluación formativa; y c) el grado de satisfacción (puntos fuertes y débiles); además de otras reflexiones generales.

De este modo, los resultados obtenidos permitirán valorar la idoneidad de la experiencia gamificada según las personas participantes, para así poder refinar en cursos posteriores cada uno de sus elementos en busca de un aumento de la motivación del alumnado universitario hacia la asignatura $y$, por ende, una mejora de los aprendizajes adquiridos en su formación inicial docente. A su vez, con la vivencia en primera persona de esta experiencia se pretende dar a conocer al futuro profesorado de EF la gamificación como un posible planteamiento didáctico en su futuro desarrollo profesional.

Finalmente, y ante el vacío científico anteriormente mencionado (Pérez-López, Rivera y Trigueros, 2017; 2019), este trabajo pretende arrojar un poco de luz al respecto para así seguir contribuyendo al desafío que comporta la innovación metodológica en la docencia del siglo XXI y, en este caso, la universitaria (FloresAguilar, 2019).

\section{MATERIAL Y MÉTODO}

\section{Sujetos}

La muestra se compone de un total de 76 estudiantes (68 chicos y 8 chicas), con un rango de edad de 21-27 años, matriculados en el tercer curso del Grado de Ciencias de la Actividad Física y del Deporte de una Universidad española, que accedieron a participar de forma voluntaria. Todos cursaban la asignatura obligatoria de Didáctica de la EF II (6 créditos) durante dos días a la semana (3 horas semanales) del segundo semestre del curso 2017/2018 (18 semanas en total). Ninguno había experimentado la Gamificación con anterioridad. Por el contrario, el docente participante era un experto en Gamificación, ya que tenía 
una sólida formación en esta metodología y había llevado a cabo experiencias similares en contextos universitarios antes del comienzo de esta investigación. No obstante, nunca había impartido clase a este grupo de estudiantes. Se empleó un diseño de investigación ex-post facto prospectivo de carácter transversal simple (Montero y León, 2007), donde un grupo clave de participantes, que posee unas características específicas (en este caso, cursar la asignatura mencionada) es seleccionado como objeto de estudio para obtener información en un momento concreto (en este caso, al finalizar el programa de intervención).

\section{Procedimiento}

Durante la primera sesión del curso se explicó a todos los alumnos matriculados que la metodología a emplear para la modalidad presencial de la asignatura iba a estar basada en la Gamificación. Se les expuso un video explicativo inicial y se les planteo la posibilidad de no participar en este planteamiento y cursar la asignatura de forma no-presencial dentro de un formato tradicional (i.e., apuntes, trabajos y examen final). Incluso a lo largo de la experiencia se mantuvo esta posibilidad de cambio en la forma de cursar la asignatura. Algunos podrían considerar que este procedimiento para implicar a los estudiantes en la investigación podría conllevar dilemas de índole ético, pero la posibilidad de no participar o abandonar la investigación en cualquier momento y cursar la asignatura de modo tradicional creemos que resuelve de manera satisfactoria estos dilemas. Por otro lado, la idea de usar todo un grupo-clase respondía a la intención de los autores de investigar los efectos de la Gamificación en un contexto natural, intacto, no modificado, para comprobar su impacto y su viabilidad de manera más real. El profesor que impartió la asignatura formaba a su vez parte del equipo investigador.

La íntegra implementación de la asignatura se basó en una experiencia educativa centrada en la hibridación de la gamificación y el AC, en el marco de la evaluación formativa, denominada "Súper Mario Bros". Cabe aclarar que dicha experiencia consistió en un cambio metodológico importante dentro de la estructura de la asignatura, ya que hasta ese momento se había impartido con metodología tradicional (i.e., clases magistrales, apuntes escritos, trabajos en grupo, evaluación fina), pero que no alteraba la enseñanza de los contenidos. De hecho, las competencias y los resultados de aprendizaje asignados fueron fielmente abordados. En la tabla 1 se sintetizan las particularidades más íntimas de "Súper Mario Bros", utilizando como hilo conductor algunos de los elementos del juego descritos por Werbach y Hunter (2012). Tal y como se ha comentado anteriormente, es importante subrayar que durante los procesos de creación y desarrollo del proyecto se han utilizado los elementos básicos del AC (Johnson et al., 2013). Para una información más detallada ver Flores-Aguilar (2019).

Tabla 1. Resumen descriptivo de los ingredientes clave del proyecto "Súper Mario Bros".

\begin{tabular}{|c|c|c|}
\hline Ingredientes & Descripción & Ilustración \\
\hline $\begin{array}{c}\text { 1. Mundo- } \\
\text { Narrativa }\end{array}$ & Mítico videojuego "Súper Mario Bros" & \\
\hline
\end{tabular}




\begin{tabular}{|c|c|c|}
\hline $\begin{array}{l}\text { 2. Misión- } \\
\text { Retos }\end{array}$ & $\begin{array}{l}\text { El objetivo final del proyecto consiste en } \\
\text { intentar vencer al maléfico Bowser (rol que } \\
\text { ejerce el docente en la prueba final del } \\
\text { juego), que se encuentra en el castillo final } \\
\text { junto a la princesa Peach. Para ello será } \\
\text { necesario superar un conjunto de retos (19 } \\
\text { en total) según cada nivel. }\end{array}$ & 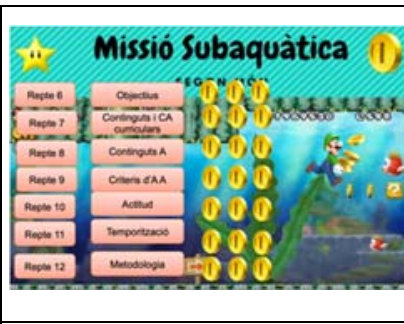 \\
\hline \multirow{3}{*}{ 3. Niveles } & \multirow{3}{*}{$\begin{array}{l}\text { Basándonos en el juego oficial, en este } \\
\text { proyecto se han generado tres niveles o } \\
\text { fases antes de la batalla final. Cada nivel } \\
\text { corresponde a un tema de la asignatura y } \\
\text { todos y cada uno de los retos a superar son } \\
\text { actividades propias de la misma } \\
\text { (relacionadas con el temario y los resultados } \\
\text { de la asignatura). }\end{array}$} & \\
\hline & & .1.Missio Subaquatica 1) \\
\hline & & . Missió fantasma \\
\hline $\begin{array}{l}4-5 . \\
\text { Avatares, } \\
\text { jugadores y } \\
\text { equipos }\end{array}$ & $\begin{array}{l}\text { Siguiendo las características del AC, se } \\
\text { crearon grupos pequeños y heterogéneos } \\
\text { (género y calificaciones de Didáctica I). Cada } \\
\text { grupo correspondía a un personaje del juego, } \\
\text { el cual debían personalizar mediante una } \\
\text { ficha, que les serviría únicamente } \\
\text { identificarse y colocarse en el tablero. }\end{array}$ & ial \\
\hline $\begin{array}{c}6 . \\
\text { Recompensas }\end{array}$ & $\begin{array}{l}\text { Monedas: al finalizar cada reto se otorgaban, } \\
\text { en función de la idoneidad de las tareas. } \\
\text { Para pasar de nivel se requerían un número } \\
\text { mínimo de monedas. En caso de no adquirir } \\
\text { las necesarias, se ampliaban las semanas } \\
\text { para la superación de los retos (correcciones } \\
\text { de los trabajos) } \\
\text { Llaves secretas: al acabar un nivel se } \\
\text { otorgaban llaves secretas (tenían un } \\
\text { mensaje con tinta invisible) que permitían la } \\
\text { entrada a un nuevo nivel y a la batalla final. }\end{array}$ & 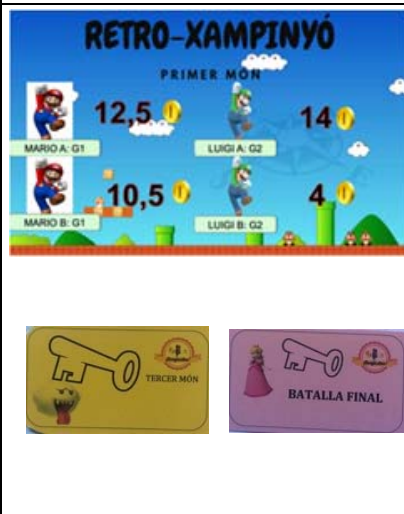 \\
\hline $\begin{array}{c}7-8 \text {. } \\
\text { Puntos de } \\
\text { experiencia y } \\
\text { bienes }\end{array}$ & $\begin{array}{l}\text { Con cada actividad extra (no obligatoria) } \\
\text { realizada se obtenían estrellas (hasta tres en } \\
\text { cada nivel). Tres estrellas se convertían en } \\
\text { una carta sorpresa con beneficios exclusivos } \\
\text { que podían utilizarse en la prueba escrita }\end{array}$ & 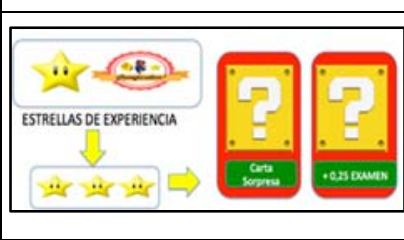 \\
\hline 9. Clasificación & $\begin{array}{l}\text { Visualización de los jugadores y los niveles } \\
\text { en el aula a través de un mural (tablero). No } \\
\text { servía para destacar a vencedores o } \\
\text { derrotados, sino para que cada jugador se } \\
\text { ubicara en el nivel donde se encontraba } \\
\text { durante el desarrollo del juego. }\end{array}$ & 9. CLAS \\
\hline $\begin{array}{l}\text { 10. Eventos } \\
\text { especiales }\end{array}$ & $\begin{array}{l}\text { Cinco eventos en total: búsqueda de } \\
\text { estrellas por el campus, kahoots sorpresas, } \\
\text { pausas activas, reto de la pirámide (batalla } \\
\text { entre equipos) y Breakout-Edu. }\end{array}$ & \\
\hline 11. Medallas & $\begin{array}{l}\text { Se entregaron premios finales a los equipos } \\
\text { que consiguieron salvar a la princesa, pero } \\
\text { también a todos los participantes. El último }\end{array}$ & $\begin{array}{l}\text { "I Certificate (I) } \\
\text { Equip MArio B }\end{array}$ \\
\hline
\end{tabular}


día de clase se simuló la entrega de premios

(trofeos de cartón) y se entregaron unos

diplomas acreditativos.

\section{Instrumentos}

\section{Cuestionario}

El equipo investigador elaboró un cuestionario ad hoc para valorar la experiencia compuesto por ocho subescalas con un total de 41 ítems y preguntas relativas a la edad, el género o el rol docente. La primera, compuesta por cuatro ítems, fue diseñada para valorar la motivación hacia la asignatura (i.e., ¿Crees que la experiencia Mario Bros ha influido positivamente en tu motivación hacia la asignatura?). La segunda, de tres ítems, preguntaba por el compromiso hacia la asignatura (i.e., ¿Crees que el proyecto Mario Bros ha influido positivamente en tu compromiso hacia esta asignatura?). La tercera, de tres ítems, preguntaba por el rendimiento académico (i.e., ¿Crees que el proyecto Mario Bros ha influido positivamente en tu rendimiento académico en esta asignatura?). La cuarta, de tres ítems, preguntaba por la consolidación de los aprendizajes (i.e., ¿Crees que el proyecto Mario Bros te ha ayudado a consolidar los aprendizajes de esta asignatura?). La quinta, de nueve ítems, preguntaba por la idoneidad de diferentes elementos de la gamificación (i.e., Valora la idoneidad-importancia de los siguientes elementos clave de la gamificación utilizados en el proyecto Mario Bros: misiones/niveles, etc.). La sexta, de dos ítems, preguntaba sobre el uso de las herramientas TIC (i.e., Valora la idoneidad-importancia de los siguientes elementos TIC utilizados: Classdojo). La séptima, de seis ítems, preguntaba sobre los cinco elementos del aprendizaje cooperativo (i.e., Valora el grado de presencia de los elementos claves para el Aprendizaje Cooperativo de Johnson et al. (2013) en tu grupo de trabajo: Responsabilidad individual, etc.). Finalmente, la octava, de 11 ítems, preguntaba sobre la evaluación formativa (i.e., Valora la utilidad-importancia de los siguientes elementos evaluadores presentes en este proyecto: autoevaluaciones de cada reto, etc.). Cada ítem fue valorado mediante una escala Likert de cuatro puntos (uno $=$ nada, dos $=$ poco, tres $=$ bastante, cuatro $=$ mucho $)$ para expresar el grado de identificación con cada una de esas preguntas. La fiabilidad de las distintas subescalas del cuestionario puede considerarse alta, que es indicativo de una alta consistencia interna; es decir, las diferentes preguntas de cada subescala estaban relacionadas entre sí y podrían contribuir a una puntuación única: "motivación hacia la asignatura" $=.697$, "compromiso hacia la asignatura $=.775$, "rendimiento académico" = .811, "consolidación de los aprendizajes" = .861, "idoneidad de los elementos de la gamificación" = .880, "uso de herramientas TIC" = .540, "aprendizaje cooperativo" $=.795$ y "evaluación formativa" $=.871$. Tan solo la subescala "uso de herramientas" TIC obtuvo un alpha de Crombach inadecuado, quizá porque solo contenía dos ítems (Vincent, 2005).

\section{Preguntas abiertas}

Al final del cuestionario se incluyeron tres preguntas abiertas para obtener información cualitativa del impacto del programa de intervención: a) Destaca algún punto débil del proyecto que acabas de experimentar; b) Destaca algún 
punto fuerte del proyecto que acabas de experimentar; y c) Expresa tus sensaciones, ideas, pensamientos entorno a la experiencia que acabas de experimentar.

Ambos instrumentos (cuestionario y preguntas abiertas) fueron elaborados online para una administración más fácil. En la última sesión, los alumnos participantes fueron requeridos por el docente para contestar en ese mismo momento al cuestionario a través de sus dispositivos electrónicos (i.e., móvil, tablet, ordenador...). Se les garantizó el anonimato y la confidencialidad de sus respuestas y se les animó a ser totalmente honestos, ya que no iba a influir en la evaluación de la asignatura cursada. Se les indicó que podían no participar en este proceso, pero que sus opiniones eran muy valiosas.

\section{Análisis de los datos}

Todos los datos cuantitativos obtenidos a través del cuestionario on-line fueron analizados mediante el programa informático SPSS, versión 22. En primer lugar, se calculó la consistencia interna de cada una de las ocho subescalas que conformaban el cuestionario a través de la obtención del alpha de Crombach. Posteriormente se realizaron análisis descriptivos de las diferentes variables estudiadas.

Los datos cualitativos obtenidos de las preguntas abiertas fueron analizados con la ayuda del software NVivo (doceava edición), a partir del cual se codificaron las principales palabras o temáticas clave, para posteriormente realizar un recuento de las frecuencias de aparición. La información obtenida fue tratada por el investigador principal usando los métodos de comparaciones constantes (Guba y Lincoln, 1994) y de inducción analítica (Patton, 1990) con el objeto de identificar y extraer categorías y patrones de respuesta comunes.

\section{RESULTADOS}

\section{Cuantitativos}

Primeramente, se obtuvieron las medias y las desviaciones estándar de cada subescala (tabla 2). Es de destacar que en todas ellas se obtuvieron valores muy próximos al máximo (4), por lo que se puede considerar la experiencia como muy bien valorada en todos los aspectos señalados.

Tabla 2. Medias y desviaciones estándar de cada subescala.

\begin{tabular}{|l|c|c|}
\hline & Media & Desviación estándar \\
\hline Motivación Asignatura & 3,17 &, 48 \\
\hline Compromiso Asignatura & 3,22 &, 55 \\
\hline Rendimiento Académico & 3,09 &, 63 \\
\hline Consolidación Aprendizaje & 3,06 &, 64 \\
\hline Idoneidad Gamificación & 3,19 &, 51 \\
\hline Uso herramientas TIC & 3,34 &, 54 \\
\hline Aprendizaje Cooperativo & 3,22 &, 42 \\
\hline Evaluación Formativa & 3,31 &, 43 \\
\hline
\end{tabular}


Por otro lado, también se solicitó a los participantes que valorasen diferentes aspectos de manera separada: rol del docente $=3.58 \pm .52$, importancia para la educación física de secundaria $=3.54 \pm .55$, satisfacción general $=3.33 \pm .59$ y formación de grupos $=2.66 \pm .96$. Nuevamente, cabe señalar los altos valores obtenidos (muy próximos al máximo valor que es cuatro), excepto en la formación de grupos. También se les pidió que pusieran nota a la asignatura (de cero a 10), obteniéndose un valor alto: $8.08 \pm 1.13$. Finalmente, se analizaron todos los datos en función del género, pero no se obtuvieron resultados significativos.

\section{Cualitativos}

El grado de satisfacción del alunando se estudió a partir de la descripción de un conjunto de puntos fuertes y débiles. En cuanto a los puntos fuertes de la experiencia, el "aumento de la motivación hacia la asignatura" ha sido el más comentado por el alumnado, seguido por las "características innovadoras de la experiencia" y por el "trabajo en equipo". El "aumento del grado de compromiso e implicación individual y grupal" también ha sido destacado, al igual que el "rol del docente" y la "narrativa escogida" (el videojuego Mario Bros). Para tratar de dar más objetividad a estos resultados, se presentan con el número de citas (extractos de texto) que se incluye en cada una de las palabras - temáticas clave obtenidas en el análisis (se presentan por ranking de aparición) (tabla 3).

Tabla 3. Puntos fuertes del proyecto según el alumnado y frecuencia de aparición.

\begin{tabular}{|c|c|c|c|}
\hline \multirow{7}{*}{ 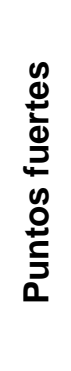 } & Palabras - temáticas clave & $\begin{array}{l}\text { Número } \\
\text { citas }\end{array}$ & $\begin{array}{l}\text { Frecuencia de } \\
\text { aparición }\end{array}$ \\
\hline & Aumento de la motivación & 21 & $27 \%$ \\
\hline & Innovación de la experiencia & 12 & $15,7 \%$ \\
\hline & Trabajo en equipo & 6 & $7,9 \%$ \\
\hline & Aumento del compromiso-implicación & 6 & $7,9 \%$ \\
\hline & Rol del docente & 4 & $5,2 \%$ \\
\hline & Narrativa: Súper Mario Bros & 3 & $4 \%$ \\
\hline
\end{tabular}

A continuación, algunas citas literales del alumnado que sirven para ejemplificar los puntos fuertes según el alumnado:

"La experiencia mantiene al alumnado motivado e implicado en todo momento" (Motivación, Pablo).

"La motivación y las ganas de aprender con la gamificación. Esto te hace partícipe del proceso de aprendizaje de forma entretenida y divertida" (Motivación-Implicación, Lourdes).

"El grado de motivación para llegar al último nivel es máximo. Esto puede ayudar para motivar a los chicos que no estén motivados para estudiar" (Motivación-Implicación. Kevin).

"El planteamiento general de la experiencia, es innovadora y original" (Innovación, Sam).

"El trabajo cooperativo y las ganas de trabajar juntos para conseguir estrellas y monedas" (Trabajo en equipo, Sasha). 
Entre los puntos débiles extraídos del análisis de la información también se destaca uno por encima del resto: "la formación de grupos heterogéneos". El alumnado alude a la presencia de compañeros y/o compañeras poco motivados o implicados, y el esfuerzo que esto les ha generado a la hora de seguir correctamente el proceso. La segunda categoría más nombrada corresponde a la "no presencia" de puntos débiles, y la tercera y la cuarta al "aumento o exceso de trabajo" para el alumnado y a la "confusión general" de algunas personas, en este orden. Igualmente, parece ser que la experiencia también ha "durado poco" para algunos estudiantes. Tal y como se ha hecho con los puntos fuertes, para tratar de dar más objetividad a estos resultados, se presentan con el número de citas (extractos de texto) que se incluye en cada una de las palabras - temáticas clave obtenidas en el análisis (se presentan por ranking de aparición) (tabla 4).

Tabla 4. Puntos débiles del proyecto y frecuencia de aparición.

\begin{tabular}{|c|c|c|c|}
\hline \multirow{6}{*}{ 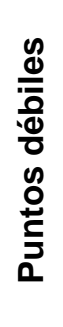 } & Palabras - temáticas clave & $\begin{array}{c}\text { Número } \\
\text { citas }\end{array}$ & $\begin{array}{c}\text { Frecuencia } \\
\text { de aparición }\end{array}$ \\
\hline & Grupos heterogéneos & 22 & $29 \%$ \\
\hline & Ninguno & 13 & $17 \%$ \\
\hline & Exceso de trabajo autónomo & 7 & $9,2 \%$ \\
\hline & Confusión general & 7 & $9,2 \%$ \\
\hline & Duración del proyecto: demasiado corto & 3 & $4 \%$ \\
\hline
\end{tabular}

De entre todas las citas literales relativas a los puntos débiles se destacan las siguientes opiniones del alumnado:

"La formación de grupos heterogéneos... a estas edades la gente que es pasota es muy pasota, y a veces perjudica el trabajo de los demás" (Grupos heterogéneos, Sonia).

"La elección de los grupos debería ser libre por parte del alumnado para poder trabajar correctamente" (Grupos heterogéneos, Antonio).

"Desde mi punto de vista, no hay un punto débil a destacar. Es original, innovador y motivador" (Ninguno, Rosa).

"La cantidad de trabajo grupal e individual en algunos momentos de la asignatura" (Exceso de trabajo autónomo, Federico).

"Al inicio era un poco dudoso, costaba seguir el hilo" (Confusión general, Luciano).

En último lugar, el alumnado también ha expresado libremente un conjunto de sensaciones, ideas, pensamientos y reflexiones personales sobre toda la experiencia vivida, las cuales se presentan en la tabla 5 también en función del número de citas (extractos de texto) que se incluyen en cada una de las palabras - temáticas clave obtenidas. 
Tabla 5. Reflexiones generales sobre la experiencia según contenido y frecuencia de aparición.

\begin{tabular}{|l|c|c|}
\hline \multicolumn{1}{|c|}{ Palabras - temáticas clave } & $\begin{array}{c}\text { Número } \\
\text { de citas }\end{array}$ & $\begin{array}{c}\text { Frecuencia } \\
\text { de aparición }\end{array}$ \\
\hline Aumento de la motivación & 25 & $33 \%$ \\
\hline Me ha gustado mucho la experiencia. & 14 & $18,4 \%$ \\
\hline Transferencia para la escuela & 9 & $12 \%$ \\
\hline Divertida & 8 & $10,5 \%$ \\
\hline Innovadora & 8 & $10,5 \%$ \\
\hline Proyecto formativo: hemos aprendido más & 5 & $6,5 \%$ \\
\hline Libre elección de grupos & 5 & $6,5 \%$ \\
\hline Nada en especial & 5 & $6,5 \%$ \\
\hline Satisfacción personal: contento/a & 4 & $5,2 \%$ \\
\hline Rol del docente: muy bueno & 3 & $4 \%$ \\
\hline Agradecimientos al docente & 3 & $4 \%$ \\
\hline
\end{tabular}

Tal y como ocurre con los anteriores puntos fuertes, el "aumento de la motivación" ha sido el comentario más repetido entre el alumnado. Además, una gran parte del alumnado valora la "experiencia como muy buena", ya que les ha "gustado mucho", e incluso afirman "sentirse contentos/as"; quizás porque se ha concebido como una experiencia "divertida, original e innovadora":

"Esta experiencia me ha hecho cambiar la visión de esta asignatura. De entrada, era una asignatura que no me despertaba motivación ni entusiasmo, pero gracias a la originalidad e innovación de este proyecto esto ha cambiado y me ha parecido muy interesante" (Motivación-innovación, Salvador).

"La experiencia me ha gustado mucho [...] Para mí, ha sido una experiencia muy divertida y motivadora que me ha permitido alcanzar a los conceptos de la asignatura" (Me ha gustado, Marc).

A su vez, "Súper Mario Bros" se valora como una "experiencia transferible a la escuela", puesto que el propio alumnado cree "haber aprendido mucho más" gracias a ella. El grupo también destaca positivamente "el rol del docente", llegando incluso a "agradecerle su trabajo":

\footnotetext{
"Me parece muy interesante utilizar este sistema como futuro docente que quiero ser" (Transferencia, Kevin).

"Es una buena idea para cambiar la enseñanza de la EF en las escuelas" (Transferencia, Sandra).

"Una nueva forma de aprender más y mejor. Muchas gracias" (Agradecimiento, Luis).

"Dar las gracias al docente por involucrarse tanto y por hacernos aprender como si estuviésemos jugando" (Agradecimiento, Magdalena).
}

En último lugar, algunas de las personas vuelven a recalcar la importancia de favorecer la "libre elección de los grupos de trabajo", mientras que otras tantas no quieren realizar "ningún tipo de comentario general" al respecto: 
"En general ha estado bien, pero creo que si los grupos los hubiésemos hecho nosotros los trabajos serían de más calidad" (Libre elección de grupos, Oriol).

\section{DISCUSIÓN}

El objetivo principal de este trabajo fue conocer la visión del alumnado participante sobre el conjunto de la experiencia gamificada "Súper Mario Bros", en base a sus propias experiencias vividas durante el transcurso de una asignatura. Más concretamente, se quiso ahondar en sus valoraciones sobre: a) los efectos de la experiencia en la motivación, el compromiso, el rendimiento y el aprendizaje; b) las características básicas de los pilares didácticos: gamificación, AC y evaluación formativa; y c) el grado de satisfacción (puntos fuertes y débiles); además de otras reflexiones generales. Tanto los resultados cuantitativos como los cualitativos han mostrado que la experiencia ha sido altamente valorada, tanto globalmente como en sus pilares identitarios como la Gamificación y sus elementos (dinámicas, mecánicas, componentes), el AC y la evaluación formativa. No obstante, también se han señalado aspectos débiles como los grupos heterogéneos, el exceso de trabajo autónomo y la confusión general.

Coincidiendo con Monguillot et al. (2015) y Pérez-López y Rivera García (2017, 2019), los resultados mostraron que la experiencia "Súper Mario Bros" aumentó el nivel de compromiso y el grado de motivación hacia la asignatura según los propios participantes; siendo esto último un aspecto muy subrayado por estas mismas personas que contradice las conclusiones de Carrasco et al. (2018) y Chan et al. (2018). En la línea de los argumentos de Kapp (2012) y Zichermann y Cunningham (2011), uno de los principales poderes de la gamificación es su efecto en la motivación de las personas participantes y los resultados obtenidos, tanto cuantitativos como cualitativos, en la presente investigación refuerzan este planteamiento.

Aunque en menor medida, los efectos de la experiencia respecto al rendimiento académico y la consolidación de los aprendizajes también han sido valorados positivamente, algo que ya señalan los trabajos sobre gamificación de Cortizo et al. (2011), Goodyear et al. (2014) y Castejón et al. (2011), respectivamente. Sin embargo, y al hilo de los argumentos de Pérez-López et al. (2017), es posible que el "peso muerto" de la mochila que contienen sus experiencias educativas, en su mayoría de carácter tradicional y directivo, no les haya permitido apreciar del todo la existencia de nuevas formas de aprender; para ello se necesitan con urgencia más metodologías innovadoras en la educación superior (Berné et al., 2011).

En esta misma tendencia, las características básicas de los pilares didácticos de "Súper Mario Bros" también han obtenido valores muy positivos. Como argumentan Melchor (2012) y Flores-Aguilar (2019), el éxito de la gamificación depende, en gran parte, de la correcta creación e implementación de sus componentes o ingredientes clave. En este caso, la narrativa, los retos y las 
misiones creadas, entre otros, parecen haber generado en el alumnado emociones positivas (alegría, diversión, felicidad, etc.). A su vez, y en la línea de los argumentos de Quintero, Jiménez y Area (2018), el uso de las TIC también ha influido positivamente en todo el proceso, sobre todo gracias a la aplicación Classdojo (Flores-Aguilar, 2019).

En cuanto al $A C$, los resultados han mostrado valores muy positivos, por lo que parece que este planteamiento metodológico ha sido bien acogido dentro de esta experiencia. No obstante, la mayoría del alumnado valora la creación de grupos heterogéneos, un requisito indispensable del AC (Velázquez, 2015), como el principal punto débil de la experiencia, aunque también aparece entre los puntos fuertes (trabajo en equipo). Como en el trabajo de Pérez-López et al. (2017), esta contradicción llama la atención, ya que siendo futuros educadores no siempre están dispuestos a compartir aprendizajes con otros estudiantes que no sean de su amistad. Quizás, esto puede ser debido a la poca experiencia y presencia previa del AC en los estudios secundarios y de educación superior (León y Latas, 2007).

Respecto a los resultados relativos a la evaluación formativa, el alumnado también ofrece una visión muy positiva al respecto. Esto permite destacar la idoneidad de su presencia en la experiencia puesto que, como señalan Boud y Falchikov (2007) y Rodríguez y Herrera (2009), su correcta implementación mejora el grado de implicación del alumnado y su aprendizaje. Como en el caso del AC, también el alumnado participante señala en relación a la evaluación formativa un punto débil: exceso de trabajo autónomo. Como se ha señalado anteriormente, el alumnado un universitario no está acostumbrado a planteamientos no-tradicionales que requieren de una implicación autónoma superior (Pérez-López et al., 2017). Son necesarias más experiencias de este tipo para que el alumnado las normalice dentro de su formación universitaria.

En definitiva, los resultados encontrados sobre los tres pilares básicos de la intervención señalados resaltan la importancia de concebir la gamificación como un modelo pedagógico que debería hibridarse con el $\mathrm{AC}$ en el marco de la evaluación formativa (Fernández-Rio y Flores-Aguilar, 2019; Flores-Aguilar, 2019).

Por otro lado, la satisfacción global del alumnado sobre la experiencia gamificada es muy elevada, hasta el punto de calificarla con un valor alto (8.08). Sobre este punto, el rol del docente adquiere un especial interés, puesto que este no ha pasado desapercibido para el alumnado; un hecho que se repite en la experiencia de Pérez-López et al. (2017) y que coincide con los ideales del docente universitario de Romero y Pérez-Ferra (2009). Por todo ello, existe una clara unanimidad entre el futuro profesorado de EF de la importancia, e incluso el deseo, de transferir este tipo de experiencias gamificadas a las sesiones de $E F$, respondiéndose así a las demandas existentes sobre la necesidad de incorporar progresivamente la gamificación en la escuela del siglo XXI (Fernández-Río y Flores-Aguilar, 2019). 


\section{CONCLUSIONES}

En términos generales, la experiencia "Súper Mario Bros" ha sido valorada positivamente por el alumnado participante, tanto a nivel de los efectos generados como en cuanto a las características estructurales de la misma.

Tal y como revela la visión del alumnado, la experiencia ha generado efectos muy deseables a nivel de compromiso, rendimiento académico y aprendizaje, pero sobre todo de motivación; gracias también a la idoneidad de sus tres pilares básicos (gamificación, AC y evaluación formativa).

Después de subrayar un gran número de puntos fuertes, el nivel de satisfacción general es tan elevado que el alumnado subraya la importancia de aumentar la presencia de este tipo de experiencias en las escuelas (transferencia), lo cual es un objetivo transversal de este trabajo, que arroja una luz de esperanza a la futura presencia de la gamificación en la educación física de la educación secundaria del futuro.

En cuanto a los problemas relativos a la formación de grupos heterogéneos, este trabajo pone de relieve la urgente necesidad de mejorar e incrementar la presencia del AC en todos los niveles educativos (primaria, secundaria y superior), pero sobre todo en la formación del profesorado. Solo de esta manera se podrán paliar las dificultades identificadas en esta experiencia.

Con todo ello, y a pesar de los riesgos que se corren cuando se apuesta por realizar un cambio metodológico de este estilo en cualquier materia, esperemos que estos resultados animen e inspiren al colectivo docente interesado.

\section{REFERENCIAS BIBLIOGRÁFICAS}

Álvarez, J. D., Grau, S. y Tortosa, M. T. (2010). Estrategias de coordinación metodológicas en la evaluación formativa de una asignatura. En C. Gómez y S. Grau (Eds.), Evaluación de aprendizajes en el EEES, 75-90. Alcoy: Marfil.

Barrientos, E. y López-Pastor, V. (2015). La evaluación formativa en educación superior. Una revisión internacional. Revista arbitrada del CIEG. 21, 272284.

Berné, C., Lozano, P. y Marzo, M. (2011). Innovación en la docencia universitaria a través de la metodología MTD. Revista de Educación, 355, 605-619.

Boud, D. y Falchikov, N. (2007). Rethinking assessment in higher education. Learning for the long term. London: Routledge. https://doi.org/10.4324/9780203964309

Carrasco, V. J., Matamoros, A. y Flores-Aguilar, G. (2019). Analysis and comparison of the results obtained after the application of a gamified methodology and a traditional one in physical education in "bachillerato" (Spanish education for 16 to 18 years old students). ESHPA -Education, Sport, Health and Physical Activity, 3(1), 29-45. 
Castejón, F., López-Pastor, V., Julián, J. y Zaragoza, J. (2011). Evaluación formativa y rendimiento académico en la formación inicial del profesorado de educación física. International Journal of Medicine and Science of Physical Activity and Sport, 11(42), 238-346.

Cortizo, J. C., Carrero, F., Monsalve, B., Velasco, A., Díaz, L. I. y Pérez, J. (2011). Gamificación y Docencia: Lo que la Universidad tiene que aprender de los Videojuegos. VIII Jornadas Internacionales de Innovación Universitaria Retos y oportunidades del desarrollo de los nuevos títulos en educación superior. Disponible en: http://abacus.universidadeuropea.es/bitstream/handle/11268/1750/46 G amificacion.pdf?sequence $=2 \& i s A l l o w e d=y$

Chan, E., Fui-Hoon, F., Liu, Q. y Lu, Z. (2018). Effect of gamification on intrinsic motivation. Springer International Publishing AG, part of Springer Nature, 445-454. https://doi.org/10.1007/978-3-319-91716-0 35

Deci, E. L. y Ryan, R. M. (1985). Intrinsic motivation and self-determination in human behavior. New York: Plenum Press. https://doi.org/10.1007/9781-4899-2271-7

Escarvajal, J. C. y Martín, F. (2019). Análisis bibliográfico de la gamificación en educación física. Revista iberoamericana de ciencias de la actividad física y del deporte,8(1), 97-109. https://doi.org/10.24310/riccafd.2019.v8i1.5770

Fernández-Río, J. (2014). Another step in models-based practice: Hybridizing Cooperative Learning and Teaching for Personal and Social Responsibility. The Journal of Physical Education, Recreation and Dance, 85(7), 3-5. https://doi.org/10.1080/07303084.2014.937158

Fernández-Río, J. y Flores-Aguilar, G. (2019). Fundamentación teórica de la Gamificación. En J. Fernández-Río (coord.). Gamificando la Educación Física. De la teoría a la práctica en Educación Primaria y Secundaria (pp.9-18). Oviedo: Ediciones Universidad de Oviedo.

Fernández-Río, J. y Méndez-Giménez, A. (2016). El aprendizaje cooperativo: modelo pedagógico para educación Física. Retos, 29, 201-2016. https://doi.org/10.47197/retos.v0i29.38721

Flores-Aguilar, G. (2016). La visión del futuro profesorado de Educación Física de Educación Secundaria sobre el aprendizaje cooperativo: conceptualización, valoración y análisis de su formación. En Actas del X Congreso Internacional de Actividades Físicas cooperativas (pp. 446459). ICE: UAB.

Flores-Aguilar, G. (2019). ¿Jugamos al Súper Mario Bros? Descripción de una experiencia gamificada en la formación del profesorado de Educación Física. Retos, 36, 529-534. https://doi.org/10.47197/retos.v36i36.67816

Flores-Aguilar, G. y Prat, M. (2018). X-Vic: corre y vuela sobre los pirineos. Un proyecto gamificado y cooperativo en educación superior. En J. Fernández-Río, A. Méndez-Giménez y R. Sánchez-Gómez (coords). Actas del XI Congreso Internacional de Actividades Físicas Cooperativas, (pp. 697-709). Oviedo: ediciones de la universidad de Oviedo.

Gallardo, F. y Carter, B. (2016). La evaluación formativa y compartida durante el prácticum en la formación inicial del profesorado: Análisis de un caso en Chile. Retos, 29, 258-263. https://doi.org/10.47197/retos.v0i29.43550 
Gibbs, G. (2003). Uso estratégico de la educación en el aprendizaje: En Brown, S. y Glasner, A. (Coord.). Evaluar en la universidad: problemas y nuevos enfoques (pp. 61-74). Madrid: Narcea.

Goodyear, V. A., Casey, A. y Kirk, D. (2014). Hiding behind the camera: Social learning within the cooperative learning model to engage girls in physical education. Sport, Education y Society, 19(6), 712-734. https://doi.org/10.1080/13573322.2012.707124

Grineski, S. (1996). Cooperative Learning in Physical Education. Champaign, IL: Human Kinetics.

Guba, E. G. y Lincoln, Y. S. (1994). Competing paradigms in qualitative research. En N. K. Denzin y Y.S. Lincoln (Eds.). Handbook of qualitative research (pp. 105-117). Thousand Oaks, CA: Sage.

Haerens, L., Kirk, D., Cardon, G. y De Bourdeaudhuij, I. (2011). Toward the development of a pedagogical model for health-based physical education. Quest, 63, 321-38. https://doi.org/10.1080/00336297.2011.10483684

Hanus, M. D. y Fox, J. (2015). Assessing the effects of gamification in the classroom: A longitudinal study on intrinsic motivation, social comparison, satisfaction, effort, and academic performance. Computers and Education, 80, 152-161. https://doi.org/10.1016/j.compedu.2014.08.019

Johnson, D. W., Johnson, R. T. y Holubec, E. J. (2013). Cooperation in the Classroom (9th ed.). Edina, MN: Interaction Book Company.

Kapp, K. M. (2012). The Gamification of learning and Instruction. San Francisco, CA: John Wiley.

Kapp, K. M.; Blair, L. y Mesch, R. (2014). The gamification of learning and instruction fieldbook. Ideas into practice. EEUU: Wiley.

Kocakoyun, S. y Ozdamli, F. (2018). "A Review of Research on Gamification Approach". En Rosalba Morese, Sara Palermo, Juri Nervo (Eds.), Socialization - A Multidimensional Perspective. https://doi.org/10.5772/intechopen.74131

León, B. y Latas, C. (2007). La formación en técnicas de aprendizaje cooperativo del profesor universitario en el contexto de la convergencia europea. Revista de Psicodidáctica, 12(2), 269-278.

León, B., Felipe, E., Iglesias, D. y Latas., C. (2011). El aprendizaje cooperativo en la formación inicial del profesorado de Educación Secundaria. Revista de Educación, 354, 715-729.

León, O., Martínez-Muñoz, L. F. y Santos-Pastor, M.L. (2019). Gamificación en educación física: un análisis sistemático de fuentes documentales. Revista iberoamericana de ciencias de la actividad física y del deporte,8(1), 110-124. https://doi.org/10.24310/riccafd.2019.v8i1.5791

López-Pastor, V. (Coord.) (2009). La Evaluación Formativa y Compartida en Educación Superior: propuestas, técnicas, instrumentos y experiencias. Madrid: Narcea.

López-Pastor, V., Pérez, A., Barba, J. y Lorente, E. (2016). Percepción del alumnado sobre la utilización de una escala graduada para la autoevaluación y coevaluación de trabajos escritos en la formación inicial del profesorado de educación física (FIPEF). CCD. Cultura Ciencia Deporte, 31, 37-50. https://doi.org/10.12800/ccd.v11i31.641 
López-Pastor, V., Pérez, D., Manrique, J. C. y Monjas, R. (2016). Los retos de la educación física en el siglo XXI. Retos, 29, 182-187. https://doi.org/10.47197/retos.v0i29.42552

Llopis, M. A. y Balaguer, P. (2016). El uso del juego en educación. Gamificación. En Chiva, O. y Martí, M. (coords) Métodos pedagógicos activos y globalizadores. Conceptualización y propuestas de aplicación (85-102). Barcelona. Graó.

Martínez-Mínguez, L. y Flores-Aguilar, G. (2014). Profesorado y egresados ante los sistemas de evaluación del alumnado en la formación inicial del maestro de educación infantil. Revista Digital de Investigación en Docencia Universitaria, 1, 29-50. https://doi.org/10.19083/ridu.8.371

Melchor, E. (2012). Gamificación y E-Learning: un ejemplo con el juego del pasapalabra. Bruselas: EFQUEL.

Monguillot, M. H., Arévalo, C. G., Mon, C. Z., Batet, L. A. y Catasús, M. G. (2015). Play the Game: gamificación y hábitos saludables en educación física. Apunts: Educación Física y Deportes, 119, 71-79. https://doi.org/10.5672/apunts.2014-0983.es.(2015/1).119.04

Montero, I., y León, O. G. (2007). A guide for naming research studies in psychology. International Journal of Clinical and Health Psychology, 7, 847-862.

Montero, E., Villalobos, J. y Valverde, A. (2007) Factores institucionales, pedagógicos, psicosociales y sociodemográficos asociados al rendimiento académico en la Universidad de Costa Rica: Un análisis multinivel. RELIEVE, 13(2), 215-234.

Orlick, T. (1982). The second cooperative sports and games book. New York, NY: Pantheon.

Palacios, A., López-Pastor, V. y Barba, J. (2013). Tipologías de profesorado universitario en función de la evaluación aplicada a los futuros docentes. Revista Estudios sobre Educación, 23, 173-195.

Patton, M. (1990). Qualitative evaluation methods (2nd ed.). Beverly Hills, CA: Sage.

Pérez-López, I. J. y Rivera García, E. (2017). Formar docentes, formar personas: análisis de los aprendizajes logrados por estudiantes universitarios desde una experiencia de gamificación. Signo y Pensamiento, 70, 9-114. https://doi.org/10.11144/Javeriana.syp36$\underline{70 . f d f p}$

Pérez-López, I. J., Rivera García, E. y Trigueros, C. (2017). «La profecía de los elegidos»: un ejemplo de gamificación aplicado a la docencia universitaria. Revista Internacional de Medicina y Ciencias de la Actividad Física y el Deporte,17(66), 243-260. https://doi.org/10.15366/rimcafd2017.66.003

Pérez-López, I. J., Rivera García, E. y Trigueros, C. (2019). 12+1. Sentimiento del alumnado universitario de educación física frente a una propuesta de gamificación: "Game of thrones: la ira de los dragones". Movimento, 25, 1-15. https://doi.org/10.22456/1982-8918.88031

Pérez-Pueyo, A. y Hortigüela, D. (2020). ¿Y si toda la innovación no es positiva en Educación Física? Reflexiones y consideraciones prácticas. Retos, 37 
Pérez-Sánchez, A. M., López-Alacid, M. P. y Poveda, P. (2009). Aprendizaje cooperativo y formación del profesor. Un estudio bibliométrico (19972008). Anales de Documentación, 12, 209-220.

Quintero, L., Jiménez, F. y Area, M. (2018). Más allá del libro de texto. La gamificación mediada con TIC como alternativa de innovación en Educación Física. Retos, 34, 343-348. https://doi.org/10.47197/retos.v0i34.65514

Rodríguez, C. y Herrera, L. (2009). Análisis correlacional-predictivo de la influencia de la asistencia a clase en el rendimiento académico universitario. Estudio de caso en una asignatura. Profesorado. Revista de currículum y formación del profesorado, 13(2), 1-13.

Romero, M. y Pérez Ferra, M. (2009). Cómo motivar a aprender en la universidad: una estrategia fundamental contra el fracaso académico en los nuevos modelos Educativos. Revista Iberoamericana de Educación, 51, 87-105. https://doi.org/10.35362/rie510628

Velázquez, C. (2015). Aprendizaje cooperativo en Educación Física: estado de la cuestión y propuesta de intervención. Retos, 28, 234-239. https://doi.org/10.47197/retos.v0i28.35533

Vincent, W. J. (2005). Statistics in Kinesiology. Champaign, IL: Human Kinetics.

Werbach, K. y Hunter, D. (2012). For the win: How game thinking can revolutionize your business. Philadelphia. Wharton Digital Press.

Número de citas totales I Total references: 53 (100\%)

Número de citas propias de la revista / Journal's own references: 1 (1,8\%)

Rev.int.med.cienc.act.fís.deporte - vol. 21 - número 83 - ISSN: 1577-0354 